\section{Randomized study of continuous high-dose lenalidomide, sequential azacitidine and lenalidomide, or azacitidine in persons 65 years and over with newly-diagnosed acute myeloid leukemia}

Bruno C. Medeiros, ${ }^{1}$ Kelly McCaul, ${ }^{2}$ Suman Kambhampati, ${ }^{3,4}$ Daniel A. Pollyea, ${ }^{5}$ Rajat Kumar, ${ }^{6}$ Lewis R. Silverman, ${ }^{7}$ Andrea Kew, ${ }^{8}$ Lalit Saini, ${ }^{9}$ CL Beach, ${ }^{10}$ Ravi Vij, ${ }^{11}$ Xiwei Wang, ${ }^{10}$ Jim Zhong, ${ }^{10}$ and Robert Peter Gale ${ }^{10,12}$

${ }^{1}$ Stanford University School of Medicine, CA, USA; ${ }^{2}$ Avera Cancer Institute, Sioux Falls, SD, USA; ${ }^{3}$ Sarah Cannon Cancer Institute at Research Medical Center, Kansas City, MO, USA; ${ }^{4}$ Kansas University Medical Center, Kansas City, KS, USA; ${ }^{5}$ University of Colorado School of Medicine Division of Hematology, Aurora, CO, USA; ${ }^{6}$ CancerCare Manitoba, Winnipeg, MB, Canada; ${ }^{7}$ Mount Sinai Hospital, New York, NY, USA; ${ }^{8}$ Queen Elizabeth II Health Sciences Center, Halifax, NS, Canada; ' University of Alberta Hospital (Adult Hematology Research), Edmonton, AB, Canada; ${ }^{10}$ Celgene Corporation, Summit, NJ, USA; ${ }^{11}$ Section of Bone Marrow Transplant and Leukemia, Washington University School of Medicine, St Louis, MO, USA and ${ }^{12}$ Imperial College London, UK

\section{ABSTRACT}

1 herapy of acute myeloid leukemia in older persons is associated with poor outcomes because of intolerance to intensive therapy, resistant disease and co-morbidities. This multi-center, randomized, open-label, phase II trial compared safety and efficacy of three therapeutic strategies in patients 65 years or over with newly-diagnosed acute myeloid leukemia: 1$)$ continuous high-dose lenalidomide $(n=15)$; 2) sequential azacitidine and lenalidomide ( $n=39)$; and 3) azacitidine only $(\mathrm{n}=34)$. The efficacy end point was 1-year survival. Median age was 76 years (range 66-87 years). Thirteen subjects (15\%) had prior myelodysplastic syndrome and $41(47 \%)$ had adverse cytogenetics. One-year survival was 21\% [95\% confidence interval (CI): 0, 43\%] with high-dose lenalidomide, 44\% (95\% CI: 28, 60\%) with sequential azacitidine and lenalidomide, and 52\% (95\%CI: 35, 70\%) with azacitidine only. Lenalidomide at a continuous high-dose schedule was poorly-tolerated resulting in a high rate of early therapy discontinuations. Hazard of death in the first four months was greatest in subjects receiving continuous high-dose lenalidomide; hazards of death thereafter were similar. These data do not favor use of continuous high-dose lenalidomide or sequential azacitidine and lenalidomide over the conventional dose and schedule of azacitidine only in patients aged 65 years or over with newly-diagnosed acute myeloid leukemia. (clinicaltrials.gov identifier: 01358734).

\section{Introduction}

The incidence of acute myeloid leukemia (AML) increases dramatically with age. Survival of older people with AML is poor, ${ }^{1-3}$ in the US, fewer than $50 \%$ of subjects with AML over 65 years of age receive therapy within three months of diagnosis despite considerable data indicating therapy improves their survival. ${ }^{4 \cdot 6}$ Reasons for not treating older patients with AML include poor performance score, co-morbidities, frailty, an antecedent hematologic disorder (AHD), presumed therapy-related leukemia, and/or adverse risk biological features. ${ }^{4,7}$ One study of more than 3000 subjects reported a significant adverse impact of increasing age on outcomes even after adjusting for these variables, suggesting age is an independent prognostic variable associated with poor outcomes.

Therapy recommendations for older subjects with AML vary with no standardof-care. ${ }^{7}$ Commonly-used treatments include intensive therapy (typically cytarabine and an anthracycline) and less intensive approaches such as low-dose cytarabine or DNA hypo-methylating drugs including azacitidine and decitabine.

\section{Haematologica 2018 \\ Volume 103(1):101-106}

\section{Correspondence:}

brunom@stanford.edu

Received: May 14, 2017.

Accepted: October 26, 2017.

Pre-published: November 2, 2017.

doi:10.3324/haematol.2017.172353

Check the online version for the most updated information on this article, online supplements, and information on authorship \& disclosures: www.haematologica.org/content/103/1/101

\section{(C)2018 Ferrata Storti Foundation}

Material published in Haematologica is covered by copyright. All rights are reserved to the Ferrata Storti Foundation. Use of published material is allowed under the following terms and conditions:

https://creativecommons.org/licenses/by-nc/4.0/legalcode. Copies of published material are allowed for personal or internal use. Sharing published material for non-commercial purposes is subject to the following conditions:

https://creativecommons.org/licenses/by-nc/4.0/legalcode, sect. 3. Reproducing and sharing published material for commercial purposes is not allowed without permission in writing from the publisher. 
Azacitidine was recently approved in Europe in subjects 65 years and over with AML and more than $30 \%$ bone marrow blasts judged unfit for a hematopoietic cell transplant. ${ }^{8}$ Approval was based on a 4-month improvement in median survival compared with conventional therapy or supportive care [10.4 vs. 6.5 months; Hazard Ratio (HR) $0.85,95 \%$ confidence interval $(95 \% \mathrm{CI}): 0.69,1.03]$.

Considerable data indicate high-dose lenalidomide, an immune-modulatory drug (IMiD $\AA$ ), may be effective in patients with myelodysplastic syndrome (MDS) with excess blasts and patients with AML judged unfit for intensive therapy. ${ }^{10,11}$ Phase I and II studies report concomitant or sequential azacitidine and lenalidomide is effective in MDS, AML and in chronic myelomonocytic leukemia (CMML). ${ }^{12-15}$

Several studies of lenalidomide used $50 \mathrm{mg} /$ day continuously without dose modification. Based on these data, we designed a randomized phase II study comparing safety and efficacy of continuous high-dose lenalidomide, sequential azacitidine and lenalidomide or azacitidine only in persons 65 years or over with newly-diagnosed AML.

\section{Methods}

We conducted a phase II randomized, open-label, parallel-group study at 25 sites in North America (clinicaltrials.gov identifier: 01358734). The study was approved by the relevant institutional review boards or an independent ethics committee, and followed guidelines set out in the Declaration of Helsinki. Subjects gave written informed consent. Statistical analyses were carried out by Celgene Corporation, Summit, NJ, USA, and were reviewed by the authors with access to all study data.

\section{Subjects}

Eligible subjects were 65 years and over with newly-diagnosed AML including those with de novo AML, AML with prior MDS or with presumed therapy-related AML. Subjects could not have previously received lenalidomide, azacitidine, decitabine or cytarabine and were judged ineligible to receive a transplant at study entry. Subjects had to have an Eastern Co-operative Oncology Group (ECOG) performance score $\leq 2$, white blood cell (WBC) count $\leq 10 \times 10^{\circ} / \mathrm{L}$ (hydroxyurea use was permitted to lower the WBC count), adequate liver and kidney function, and no uncontrolled infection or other cancer within two years of study entry. Subjects who had received prior therapy for AML other than hydroxyurea were not eligible for the study.

\section{Study design}

Screening procedures were completed no more than 28 days before randomization. Confirmation of AML for study entry was based on local pathology review. A separate central review of all bone marrow aspirates, bone marrow biopsies and blood smears was conducted by a blinded pathologist (Dr. Daniel Arber, Stanford University). Cytogenetic testing on diagnostic samples was carried out locally and reviewed centrally (Prof. Athena Cherry, Stanford University). Subjects were randomized equally to the therapy arms by central interactive voice response system (IVRS). Randomization was stratified for performance score (0-1 vs. 2) and levels of blood myeloblasts ( $<1$ vs. $\left.\geq 1 \times 10^{9} / \mathrm{L}\right)$.

\section{Therapy}

Therapy regimens are shown in Figure 1. Therapy in any arm could be interrupted or delayed because of hematologic or nonhematologic therapy-related adverse events (AEs). Lenalidomide dose-reductions were not allowed in cycles 1-4 in the continuous high-dose lenalidomide cohort or in courses 1 and 2 in the azacitidine and lenalidomide cohort. Dose reductions of lenalidomide

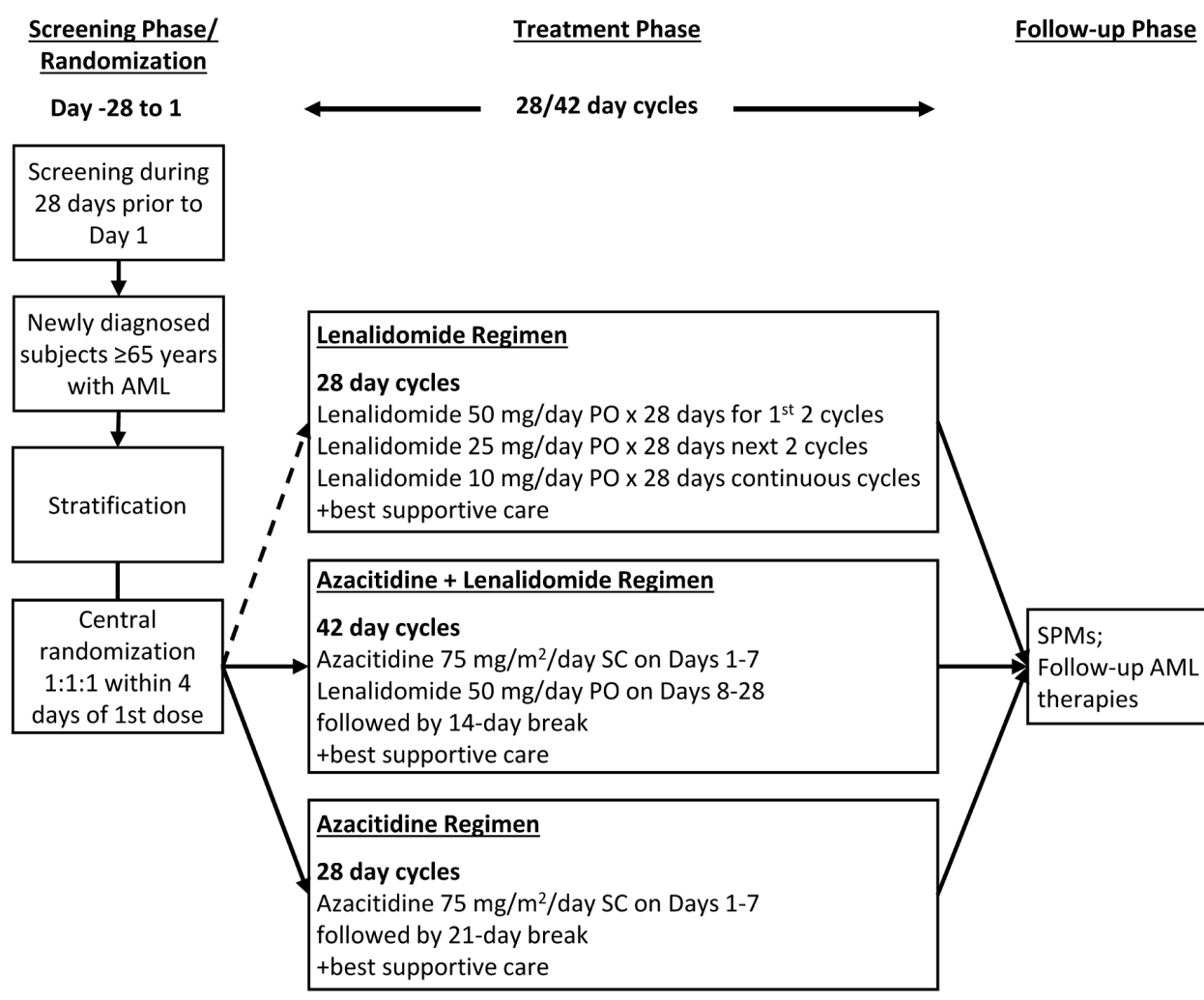

Figure 1. Study design and therapy regimens. Stratification factors were Eastern Co-operative Oncology Group (ECOG) performance score (0-1 vs. 2) and blood blast level ( $<1$ vs. $\geq 1 \times 10^{\circ} / \mathrm{L}$ ) Randomization in the lenalidomide regime was suspended $11^{\text {th }}$ September 2013 and permanently closed on $15^{\text {th }}$ April 2014 as per Amendment 2 because of the high rate of discontinuation of the study treatment in subjects receiving high-dose continuous lenalidomide. AML: acute myeloid leukemia; PO: per oral administration; SC: subcutaneous injection; SPM: secondary primary malignancies. 
could be made thereafter in both cohorts. Dose reductions of azacitidine were permitted as described. Dose reductions of lenalidomide or azacitidine were continued in subsequent cycles unless additional adverse events occurred; in such cases, additional dose reductions were permitted. Subjects were seen weekly for the first 2 courses, every two weeks for the next 2 courses, and on the first day of subsequent courses. Microscope slides of blood and bone marrow samples and cytogenetic testing were obtained pre-randomization, on day 15 of course 1 , within seven days of beginning courses 2 and 3 , and before starting every third subsequent course.

\section{Safety and efficacy end points}

Safety assessments included vital signs, physical exam, weight, ECOG performance score, complete blood count (CBC) and differential, blood chemistries, urinalyses, coagulation, thyroid function tests and electrocardiograms. AEs were recorded using Common Terminology Criteria for Adverse Events (CTCAE) v.4.0. Study subjects were kept under surveillance for the development of new cancers. Events of interest (EOIs) were recorded and reported as serious AEs (SAEs) and considered medically-important even if not meeting SAE criteria. The primary end point was 1-year survival. Secondary end points included rates of complete remission (CR), complete remission with incomplete recovery of neutrophils or platelets (CRi), and remission duration. Subjects discontinuing the study could receive subsequent therapy during the follow-up interval at their physician's discretion and with their consent.

\section{Statistical analysis}

The study was not designed or powered for a formal statistical comparison of the therapy cohorts and a sample size calculation was not made. Target enrollment was 40 subjects per cohort.

Table 1. Baseline variables for the intent-to-treat populations.

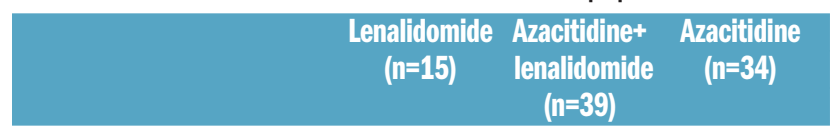

Age (years)

\begin{tabular}{lccc} 
Median (range) & $80(68-85)$ & $76(66-87)$ & $75(66-85)$ \\
$>75$ years (n) & 10 & 21 & 14 \\
Male (n) & 12 & 22 & 19 \\
\hline
\end{tabular}

ECOG performance score (n)

\begin{tabular}{lccc}
$0-1$ & 13 & 32 & 27 \\
2 & 2 & 7 & 6 \\
Prior MDS (n) & 3 & 5 & 5 \\
\hline Adverse cytogenetics (n) & 4 & 20 & 17 \\
Blood blasts >1x10E\%/L (n) & 11 & 31 & 27 \\
\hline $\begin{array}{l}\text { Bone marrow blasts (\%) } \\
\text { Median (range) }\end{array}$ & $56(22-95)$ & $37(12-84)$ & $34(14-70)$ \\
$\begin{array}{l}\text { Interval from diagnosis } \\
\text { to treatment (days) }\end{array}$ & & & \\
Median (range) & $15(7-42)$ & $18(4-41)$ & $16(6-50)$ \\
\hline $\begin{array}{l}\text { Platelets (x10/L) } \\
\text { Median (range) }\end{array}$ & $54(8-162)$ & $72(13-204)$ & $56(8-211)$ \\
Neutrophils (x10 9 L) & & & \\
Median (range) & $0.2(0-1.6)$ & $0.4(0-3)$ & $0.5(0-3)$ \\
\hline
\end{tabular}

n: number; ECOG: Eastern Co-operative Oncology Group; MDS: myelodysplastic syndromes; d: days.
Preliminary analyses indicated a high rate of discontinuations in the continuous high-dose lenalidomide cohort with 10 of the first 13 subjects receiving less than 56 days of therapy. Based on these data, the Data Safety Monitoring Committee (DSMC) recommended closing randomization into this cohort and the planned study sample size was reduced to 95 subjects.

Time-to-death from any cause was defined as the interval from randomization to death. Living subjects were censored at date of last contact, withdrawal of consent, loss to follow up, or study completion. There was no pre-specified statistical plan to compare survival between the cohorts. However, an exploratory analysis was made comparing 1-year survival Kaplan-Meier estimates between the cohorts using the log rank test and which indicated non-proportional hazards of death. Consequently, we estimated HR and $95 \%$ confidence intervals for the intervals $0-4$ months and $>4-12$ months using a Cox proportional hazards model. $P$-values were derived from the Cox model.

\section{Results}

\section{Subjects}

Enrollment began on $27^{\text {th }}$ April 2012 with database lock on $1^{\text {st }}$ May 2015. Because of poor accrual, only 88 subjects were randomized, all of whom constitute the intent-totreat (ITT) population including 15 subjects randomized to receive high-dose continuous lenalidomide, 39 randomized to receive sequential azacitidine and lenalidomide, and 34 randomized to receive azacitidine only. Subjects in the high-dose lenalidomide cohort were more likely to be over 75 years of age and to have had prior MDS, had a higher percent of bone marrow blasts, and lower levels of blood neutrophils. This cohort also had fewer subjects with adverse cytogenetics than the other cohorts (Table 1). There were 6 subjects with $\operatorname{del}(5 / 5 q)$, including one each in the high-dose lenalidomide and in the azacitidine only cohorts, and 4 in the sequential azacitidine and lenalidomide cohort.

\section{Efficacy}

Median therapy duration was six weeks (range 1-48 weeks) for the continuous high-dose lenalidomide cohort, eight weeks (0-78 weeks) for the sequential azacitidine and lenalidomide cohort, and 29 weeks (1-143 weeks) for

Table 2. Hazard Ratios for death.

\begin{tabular}{|c|c|c|}
\hline & Hazard Ratio (95\% CI) & $P$ \\
\hline \multicolumn{3}{|l|}{$0-4$ months } \\
\hline Lenalidomide $v s$. azacitidine & $5.73(1.90,17.20)$ & 0.002 \\
\hline $\begin{array}{l}\text { Azacitidine + lenalidomide } v s \text {. } \\
\text { azacitidine }\end{array}$ & $2.51(0.89,7.05)$ & 0.081 \\
\hline $\begin{array}{l}\text { Lenalidomide } v s \text {. } \\
\text { azacitidine + lenalidomide }\end{array}$ & $2.19(0.94,5.13)$ & 0.071 \\
\hline \multicolumn{3}{|l|}{$>4-12$ months } \\
\hline Lenalidomide $v$ s. azacitidine & $0.853(0.187,3.90)$ & 0.838 \\
\hline $\begin{array}{l}\text { Azacitidine + lenalidomide } v s \text {. } \\
\text { azacitidine }\end{array}$ & $0.790(0.312,2.00)$ & 0.620 \\
\hline $\begin{array}{l}\text { Lenalidomide } v \text { s. azacitidine + } \\
\text { lenalidomide }\end{array}$ & $1.02(0.22,4.80)$ & 0.982 \\
\hline
\end{tabular}

CI: Confidence Interval; $P$ : $P$-value; vs.: versus. 


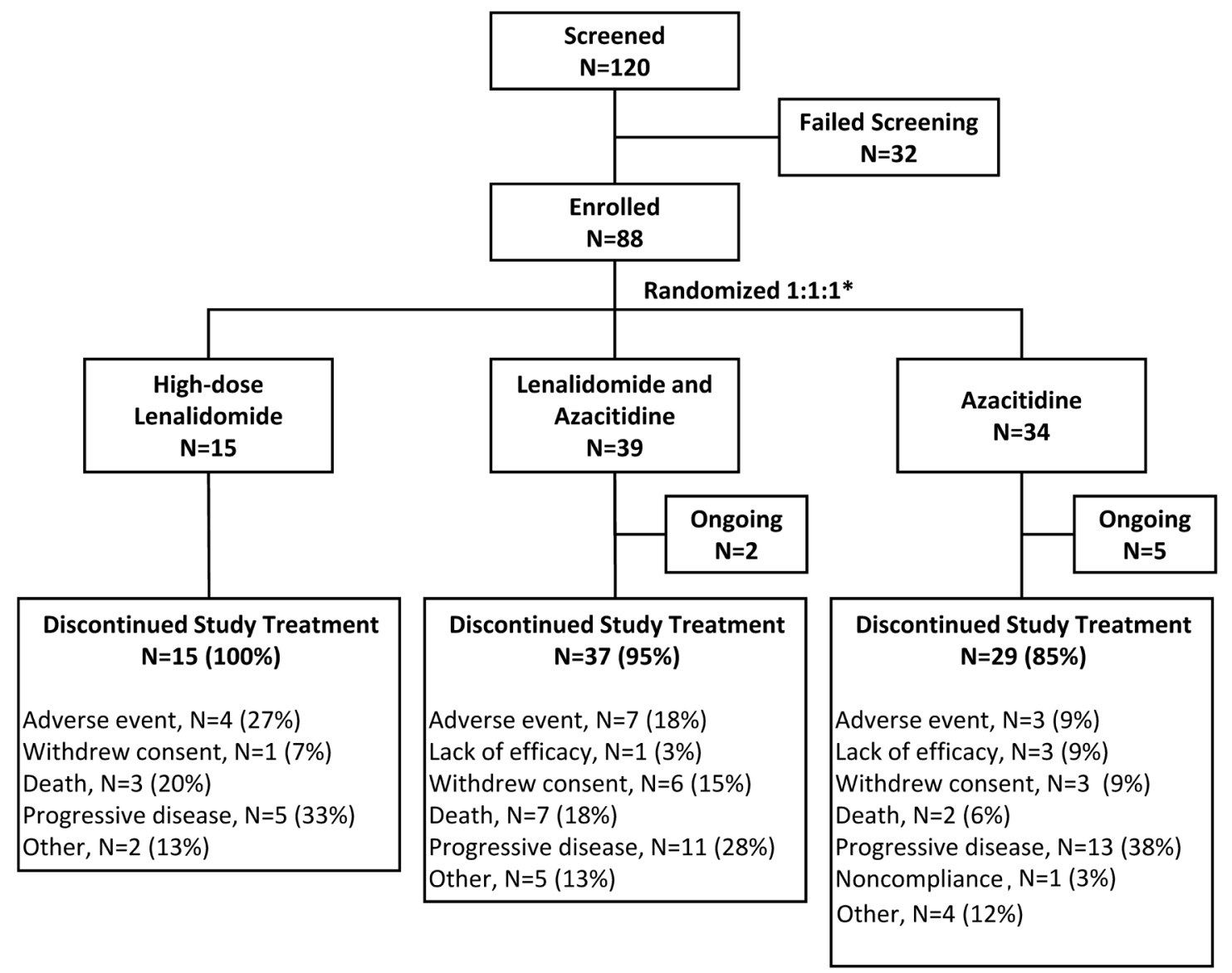

Figure 2. CONSORT study-flow diagram. *Four randomized subjects were not treated because of withdrawal of consent, adverse events, or other reasons, e.g. hospitalization. Percents are based on the intention-to-treat population.

the azacitidine only cohort. The most common reason for discontinuation was leukemia progression in 5, 11, and 13 subjects (Figure 2).

One-year survivals were $21 \%(95 \% \mathrm{CI}$ : 0, 43\%), 44\% (95\% CI: 28, 60\%) and 52\% (95\% CI: 35, 70\%) (Figure 3) in the lenalidomide, azacitidine and lenalidomide and azacitidine only cohorts. Subjects in the high-dose continuous lenalidomide cohort had a higher hazard of death than subjects in the azacitidine cohort [HR 5.73 (1.91, 17.20); $P=0.002$ ] or subjects in the sequential azacitidine and lenalidomide cohort [HR 2.19 (95\% CI: 0.94, 5.13); $P=0.071$ ] (Table 2) in the first four months post randomization. Hazard of death was also higher in the sequential azacitidine and lenalidomide cohort compared with the azacitidine cohort [HR $2.51(0.89,7.05) ; P=0.081]$. Hazards of death $>4-12$ months were similar in the 3 cohorts.

There were 7 deaths in the continuous high-dose lenalidomide cohort in the first four months. Median duration of therapy of these subjects was 36 days (range 7-84 days). Two subjects discontinued therapy because of an adverse event and 5 because of AML progression. Causes of death were AML and/or its complications such as infection. One subject had a pulmonary infarct. Rates of CR and CRi for the cohorts are shown in Table 3.
Table 3. Proportion of subjects with complete remission with (CR) and without (CRi) complete hematologic recovery.

\begin{tabular}{lccc} 
& CR & CRi & CR/CRi \\
Lenalidomide $(\mathrm{n}=15)$ & 2 & 3 & 5 \\
Azacitidine + lenalidomide $(\mathrm{n}=39)$ & 11 & 4 & 15 \\
\hline Azacitidine $(\mathrm{n}=34)$ & 6 & 8 & 14 \\
\hline
\end{tabular}

n: number

Interestingly, 2 subjects receiving high-dose lenalidomide only, a non-cytotoxic drug, achieved a complete remission and 3 , a complete remission with incomplete recovery of blood levels of neutrophils and/or platelets. There were too few subjects with del $(5 / 5 q)$ in each cohort to compare outcomes of this subset.

\section{Adverse events}

Eighty-four subjects received 1 dose or more of study drug and constitute the safety population including 14 subjects receiving high-dose continuous lenalidomide, 38 receiving sequential azacitidine and lenalidomide, and 32 receiving azacitidine only. Treatment was discontinued within the first 2 cycles in 10 subjects in the high-dose continuous lenalidomide, 19 in the sequential azacitidine and lenalidomide cohort, and 8 in the azacitidine only 
cohort. The most common reasons for discontinuation of study therapy within 2 courses across the 3 cohorts were adverse events ( 3,6 and 2 , respectively), death (2, 6 and 1 , respectively) and disease progression (3,2 and 2 , respectively). During the study the proportion of treatmentemergent adverse events (TEAEs) resulting in therapy discontinuation was highest in the high-dose continuous lenalidomide cohort $(n=4)$ followed by the azacitidine and lenalidomide cohort $(n=7)$, and lowest in the azacitidine only cohort $(n=3)$. Infections were the most frequent cause of permanent therapy discontinuation and were most frequent in the high-dose continuous lenalidomide cohort ( $\mathrm{n}=4$ compared with 3 subjects in the azacitidine and lenalidomide cohort and 1 subject in the azacitidine only cohort). There were 3 new cancers (vulvar cancer, stage 0 ; lung adenocarcinoma; and central nervous system cancer), all in the azacitidine only cohort. These cancers were diagnosed at approximately, 6, 9 and less than 1 month post randomization.

The most frequent TEAEs (any grade) considered drugrelated were hematologic and gastrointestinal. The most frequent TEAE grade 3 or over was febrile neutropenia $(n=6,17$ and 9, respectively) (Table 4). Frequencies of treatment-emergent serious AEs (TE-SAEs) was higher in the high-dose continuous lenalidomide cohort $(\mathrm{n}=10)$ than in the other cohorts ( $\mathrm{n}=16$ and 7 , respectively). Pneumonia was the most frequent infectious TE-SAE reported in 1,2 and 0 subjects, respectively. The most common causes of death on therapy were AML progression and infections. Deaths from AML progression were reported in 8,12 and 11 subjects and deaths from infections were reported in 2, 5 and 2 subjects, respectively. Median hospital days for the 3 cohorts were ten days (range 2-54 days), 11 days (range 2-43 days), and seven days (range 2-24 days).

\section{Discussion}

Our randomized phase II study in patients over 65 years with newly-diagnosed AML was designed to numerically compare 1-year survivals between the cohorts. It was not designed nor powered to make statistical comparisons in outcomes. The sequential azacitidine and lenalidomide and azacitidine only regimens were reasonably well-tolerated. Proportions of subjects alive at one year in these cohorts were $52 \%(35,70 \%)$ and $44 \%(28,60 \%)$, similar to azacitidine only in a large phase III trial in similar patients ${ }^{16}$ However, the HR for death of $2.51(0.89,7.05)$ in the lenalidomide plus azacitidine cohort in months $0-4$ suggests azacitidine only may be the best regimen. Lenalidomide at the dose and schedule used in the highdose continuous lenalidomide cohort was less well-tolerated, perhaps because no dose reduction was permitted, resulting in a high proportion of early treatment discontinuation and in subsequent leukemia progression. Subjects

Table 4. Most common ( $\geq 3$ subjects) grade $\geq 3$ treatment-emergent adverse events.

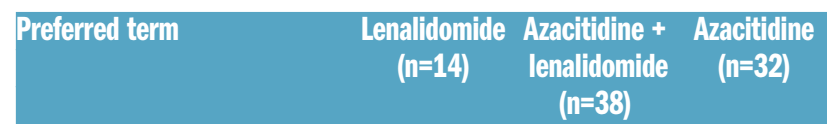

\begin{tabular}{lccc}
$\geq 1$ CTCAE $\geq$ grade-3 TEAE & 14 & 34 & 29 \\
Febrile neutropenia & 6 & 17 & 9 \\
\hline Thrombocytopenia & 4 & 12 & 11 \\
Anemia & 4 & 10 & 8 \\
\hline Neutropenia & 3 & 5 & 9 \\
Pneumonia & 3 & 2 & 8 \\
\hline Cellulitis & 0 & 5 & 4 \\
Neutrophil count decreased & 0 & 5 & 4 \\
\hline WBC decreased & 0 & 4 & 5 \\
Platelet count decreased & 0 & 6 & 2 \\
\hline Dyspnea & 2 & 4 & 1 \\
Fatigue & 4 & 6 & 5 \\
Syncope & 3 & 1 & 3 \\
Atrial fibrillation & 0 & 4 & 0 \\
\hline
\end{tabular}

CTCAE: Common Terminology Criteria for Adverse Events;TEAE: treatment-emergent adverse event;WBC: white blood cell count. Subjects with multiple occurrences of the same TEAE were counted only once and assigned the highest grade reported. Data cut-off date: $1^{\text {1s }}$ May 2015. Preferred terms are according to MedDRA dictionary, v. 17.0 and listed in descending order of overall frequency.

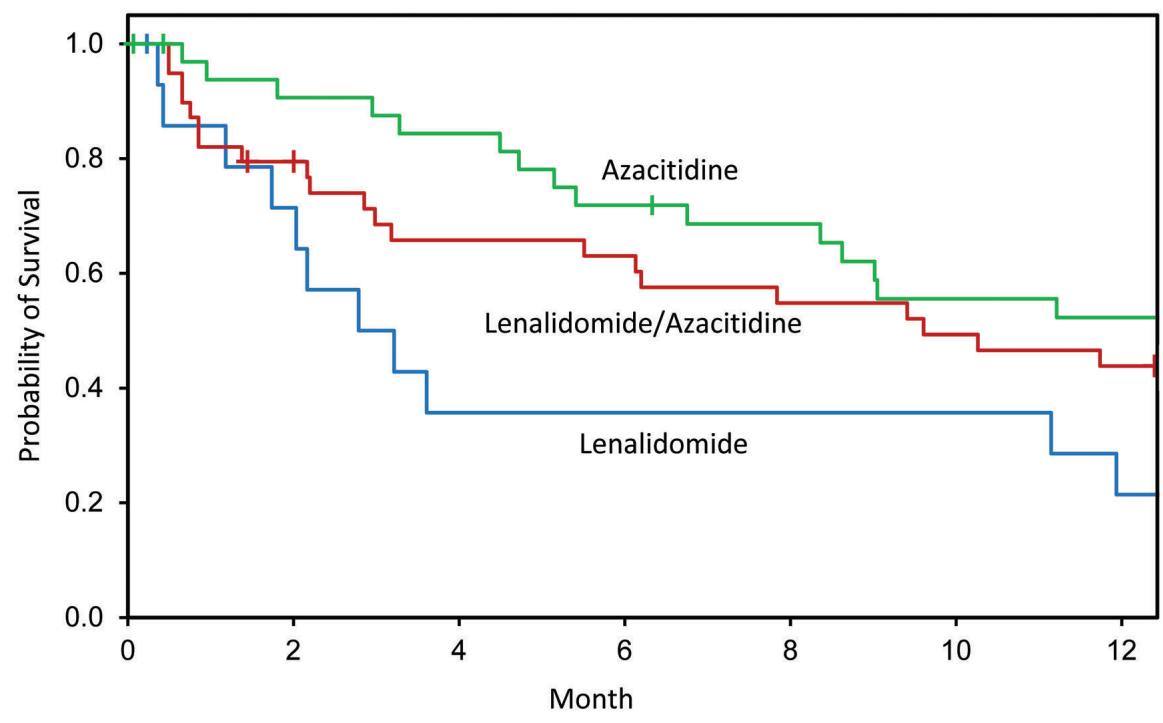

Figure 3. Kaplan-Meier estimates of 1 year survival. Subjects in the high-dose continuous lenalidomide cohort had a higher hazard of death in 0-4 months than subjects in the azacitidine alone cohort $(P=0.002)$ and subjects in the sequential azacitidine and lenalidomide cohort $(P=0.071)$. 
enrolled in this cohort had an increased hazard of death in the first four months of therapy compared with the other cohorts. Hazards of death thereafter were similar but favor the azacitidine cohort. This early difference in hazard of death should be viewed cautiously as these analyses were not specified in the pre-study statistical plan and sample sizes are small. Proportions of subjects achieving a CR/CRi were similar with wide $95 \%$ confidence intervals. It is also important to emphasize that the small sample sizes may have resulted in an imbalance in baseline variables. For example, subjects in the high-dose continuous lenalidomide cohort were older and more likely to have prior MDS and severe bone marrow failure but less frequent adverse cytogenetics. This cohort also had a greater proportion of subjects with cardio-vascular disease risk factors (data not shown). None of these differences were tested for statistical significance according to the prestudy statistical plan. Finally, discontinuation rates were generally similar to those in other clinical studies of continuous high-dose lenalidomide in older persons with AML. ${ }^{14}$ Randomization with or without stratification does not guarantee cohorts have similar distributions of known and latent prognostic variables, especially when there are few subjects in each cohort. Typically, potential imbalances are accounted for by calculating $P$-values and indicating confidence intervals. Because we did not perform these calculations for outcomes other than for 0-4 and $>4$ 12 -month survival intervals between the cohorts, differences in outcomes should not be assumed to result from therapy assignment.

Sequential azacitidine and lenalidomide did not increase the frequency of $>$ grade 3 or over TEAEs compared with continuous high-dose lenalidomide. Subjects in the continuous high-dose lenalidomide cohort were scheduled to receive $50 \mathrm{mg} /$ day for 56 days whereas those in the sequential azacitidine and lenalidomide cohort were scheduled to receive the same dose of lenalidomide for 21 days followed by 14 days off therapy. Consequently, there are significant differences in schedule and total dose of lenalidomide between the cohorts. Neither regimen with high-dose lenalidomide, continuous or sequential, was as well-tolerated as azacitidine only.

In conclusion, data from unplanned survival analysis indicate high-dose continuous lenalidomide given without dose reduction resulted in a high rate of early discontinuations and an early but not late increased hazard of death compared with sequential azacitidine and lenalidomide and with azacitidine only in subjects 65 years and over with newly-diagnosed AML. Our data also suggest therapy with azacitidine only may be better than sequential lenalidomide and azacitidine based on the 95\% confidence interval of the HR for death. Although continuous high-dose lenalidomide is active in AML and sometimes produces prolonged complete remissions,$^{10}$ we cannot recommend the dose and schedule we tested for subjects 65 years and over with newly-diagnosed AML. Adding lenalidomide to azacitidine did not improve 1-year survival and may have reduced it in the first four months of therapy. However, our study was neither designed nor powered to test this question. Whether a different dose, schedule and/or dose-modification of lenalidomide alone or with azacitidine might be better tolerated and/or more effective than the dose and schedule we studied is unknown. However, the achievement of complete remission with or without complete recovery of blood levels of neutrophils and/or platelets by a non-cytotoxic drug is noteworthy.

\section{Acknowledgments}

Medical Communication Company (Wynnewood, PA, USA) provided editorial support in the form of arranging references for an advanced draft which was subsequently approved by all authors.

\section{Funding}

The study was funded by Celgene Corporation.

\section{References}

1. Siegel R, Naishadham D, Jemal A. Cancer statistics, 2013. CA Cancer J Clin. 2013;63(1):11-30.

2. Appelbaum FR, Gundacker H, Head DR, et al. Age and acute myeloid leukemia. Blood. 2006;107(9):3481-3485.

3. Buchner T, Krug UO, Gale RP, et al. Age, not therapy intensity, determines outcomes of adults with acute myeloid leukemia. Leukemia. 2016;30(8):1781-1784.

4. Medeiros BC, Satram-Hoang S, Hurst D, Hoang KQ, Momin F, Reyes C. Big data analysis of treatment patterns and outcomes among elderly acute myeloid leukemia patients in the United States. Ann Hematol. 2015;94(7):1127-1138.

5. Juliusson G. Older patients with acute myeloid leukemia benefit from intensive chemotherapy: an update from the Swedish Acute Leukemia Registry. Clin Lymphoma Myeloma Leuk. 2011;11 (Suppl 1):S54-S59.

6. Juliusson G. Most 70- to 79-year-old patients with acute myeloid leukemia do benefit from intensive treatment. Blood. 2011;117(12):3473-3474
7. National Comprehensive Cancer Network. Clinical Practice Guidelines in Oncology. Acute Myeloid Leukemia, version 1.2017, Available at: http://www.nccn.org/professionals/physician_gls/f_guidelines.asp.

8. Krug U, Berdel WE, Gale RP, et al. Increasing intensity of therapies assigned at diagnosis does not improve survival of adults with acute myeloid leukemia. Leukemia. 2016;30(6):1230-1236.

9. National Comprehensive Cancer Network (NCCN) Clinical Practice Guidelines in Oncology. Acute Myeloid Leukemia. Version 2.2014, Available at: http://www.nccn.org/professionals/physician_gls/f_guidelines.asp; 2014 [Last accessed $5^{\text {th }}$ August 2014]

10. Fehniger TA, Uy GL, Trinkaus K, et al. A phase 2 study of high-dose lenalidomide as initial therapy for older patients with acute myeloid leukemia. Blood. 2011; 117(6):1828-1833.

11. Pollyea DA, Zehnder J, Coutre S, et al. Sequential azacitidine plus lenalidomide combination for elderly patients with untreated acute myeloid leukemia. Haematologica. 2013;98(4):591-596.

12. Ramsingh G, Westervelt P, Cashen AF, et al.
A phase 1 study of concomitant high-dose lenalidomide and 5 -azacitidine induction in the treatment of AML. Leukemia. 2013;27(3):725-728.

13. Wei A, Tan P, Perruzza S, et al. Maintenance lenalidomide in combination with 5-azacitidine as post-remission therapy for acute myeloid leukaemia. Br J Haematol. 2015; 169(2):199-210.

14. Sekeres MA, Gundacker H, Lancet J, et al. A phase 2 study of lenalidomide monotherapy in patients with deletion $5 q$ acute myeloid leukemia: Southwest Oncology Group Study S0605. Blood. 2011; 118(3):523-528.

15. Sekeres M, Othus M, List AF, et al. Randomized phase II study of azacitidine alone or in combination with lenalidomide or with vorinostat in higher-risk myelodysplastic syndromes and chronic myelomonocytic leukemia: North American Intergroup Study SWOG S1117. J Clin Oncol. 2017;35(24):2745-2753.

16. Dombret H, Seymour JF, Butrym A, et al. International phase 3 study of azacitidine vs conventional care regimens in older patients with newly diagnosed AML with $>30 \%$ blasts. Blood. 2015;126(3):291-299. 